CASE REPORT

\title{
New World leishmaniasis from Spain
}

\author{
N Tomson, R P Symonds, A A Moir, C H Kendall, M J Wiselka
}

Postgrad Med J 2002;78:757-758

A 69 year old man living in Spain contracted mucocutaneous leishmaniasis involving the nose. The infecting organism was Leishmania infantum, which only rarely causes the New World form of the disease. The source of infection was probably a neighbour's dog. The patient began treatment with liposomal amphotericin B but died of pneumonia two months later.

I

nfection, inflammatory disease, and neoplasia are possible causes of a chronically painful red swollen nose. In Britain neoplasia is the diagnosis most likely to be considered first. (see box 1). Most of the bacterial and fungal causes are rare and usually affect other parts of the body as well as the nasal passages. The same is also true of the protozoal infection leishmaniasis. Involvement of the nose in this disease is extremely rare outside Central and South America.

\section{CASE REPORT}

A 69 year old British expatriate returned from Malaga in Spain where he had lived for the last five years. He complained of nasal obstruction, crusting with a mucopurulent discharge, pain, hyposmia, and occasional epistaxis. On examination his nose was red and swollen (see fig 1) with granular mucosa in the left nasal vestibule. The nose was very tender preventing further examination. He was apyrexial. Systemic examination did not reveal any further cutaneous lesions, enlarged lymph nodes, or hepatosplenomegaly. Similarly the full blood count, urea and electrolytes, liver function tests, and chest radiograph were within normal limits.

\section{Box 1: Cause of chronic painful ulceration of nasal \\ mucosa}

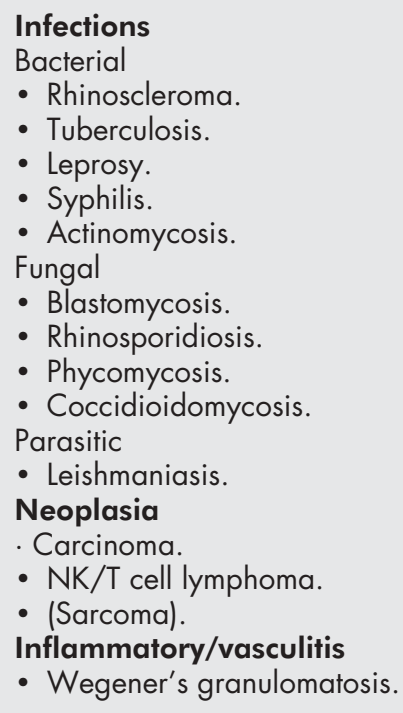

Infections

Bacterial

- Rhinoscleroma.

- Tuberculosis.

- Leprosy.

- Syphilis.

- Actinomycosis.

Fungal

- Blastomycosis.

- Rhinosporidiosis.

- Phycomycosis.

- Coccidioidomycosis.

Parasitic

- Leishmaniasis.

Neoplasia

Carcinoma.

- NK/T cell lymphoma.

- (Sarcoma).

Inflammatory/vasculitis

- Wegener's granulomatosis.

Computed tomography of the paranasal sinuses showed only mucosal hypertrophy with a possible soft tissue mass in the inferior part of the nasal septum but no evidence of bony destruction. Cultures of a nasal swab grew Staphylococcus aureus and oral flucloxacillin was prescribed.

An examination under anaesthetic showed no discrete mass but a biopsy of the nasal septum revealed inflammatory cells, macrophages, and mature B and T lymphocytes. Many of the macrophages contained intracytoplasmic parasites which on staining with Giemsa were identified as amastigotes of Leishmania spp (fig 2). Using polymerase chain reaction amplification the infecting organism was identified as Leishmania infantum. The serum leishmania direct agglutination test, agglutination, and K-39 antibody tests were negative. Bone marrow aspirate and trephine showed no evidence of any dissemination of leishmaniasis.

At this point the patient discovered his neighbour's dog (in Malaga) was suffering from leishmaniasis and the veterinary surgeon treating the animal confirmed that canine infection with leishmania was very common in Malaga.

The patient began treatment with liposomal amphotericin B. He received two weeks of treatment at a dose of $3 \mathrm{mg} / \mathrm{kg}$ with partial regression of the lesion. He relapsed as soon as

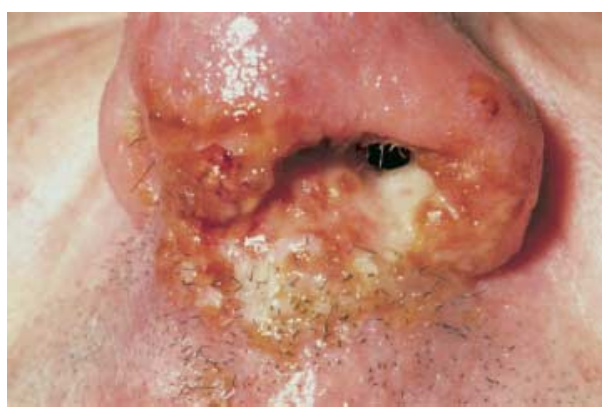

Figure 1 Nasal lesion before treatment.

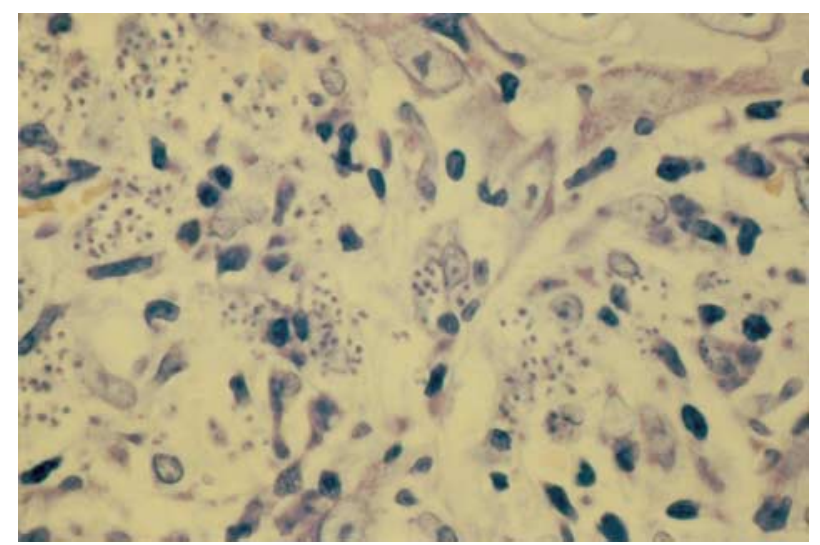

Figure 2 Histology of nasal lesion showing swollen macrophages containing numerous leishmania amastigotes; Giemsa stain (original magnification $\times 1000$ ). 
treatment was discontinued and had a further two courses of treatment. He failed to respond to treatment and died two months after presentation with renal impairment and nosocomial pneumonia. Further investigations for immunodeficiency, including HIV testing were all negative.

\section{DISCUSSION}

Infection by protozoa haemoflagates of the genus leishmania can cause a wide range of clinical syndromes which are grouped together as visceral, cutaneous, or mucocutaneous disease. ${ }^{1}$ Imported leishmania infection is on the increase as a result of increased travel to endemic areas and adventure tourism.

There are at least 30 species of leishmania of which 12 named and several un-named affect man. ${ }^{2}$ The protozoa are intracellular (amastigote) in rodents and dogs, which act as a reservoir, and there is an extracellular form (promastigote) in sandflies, which are the vector.

The mucocutaneous form of the disease is almost exclusively restricted to South America where it is known as espundia. Untreated, this can lead to gross nasal oral and laryngopharyngeal destruction and death. All reported cases in the UK have been in travellers from South America. ${ }^{34}$ The protozoon responsible is commonly $L$ braziliensis, and rarely $L$ panamensis or L guyanesis, which are only found in South America.

Infection in the Mediterranean is usually caused by $L$ infantum, leading to visceral and less commonly cutaneous lesions, usually in children under 5. In 1990 the World Health Organisation stated that mucocutaneous infection due to $L$ infantum was unknown. ${ }^{1}$ However, two cases of midfacial granuloma caused by leishmania were reported from France in $1990^{5}$ and in 1991 a case of lingual and palatine infection by $L$ infantum was described in a 60 year old man from Sardinia. ${ }^{6}$ The incidence of $L$ infantum infection in Southern Europe has increased over recent years, largely resulting from HIV coinfection; however, even in this group of patients mucocutaneous disease is very rare. ${ }^{7}$

The reservoir for infection in Southern Spain is the dog and over three quarters of dogs in Malaga show signs of infection (personal communication, 1999). As this case illustrates Mediterranean subtypes can rarely cause the "New World" forms of this disease. Southern Spain is a very common British tourist destination. The risk of developing leishmaniasis is low but other similar cases have occurred (personal communication, Liverpool School of Tropical Medicine).

The treatment options for invasive leishmania infection include pentavalent antimonials in a conventional treatment regimen of $20 \mathrm{mg}$ antimony $/ \mathrm{kg} / \mathrm{day}$ for 30 days, paromomycin (which may be combined with antimonials), and amphotericin B in conventional or liposomal formulations. ${ }^{89}$ Resistance to antimonials is becoming an increasing problem, particularly for $L$ donovani, but remains rare in $L$ infantum. This patient was treated with liposomal amphotericin $B$ which is

\section{Clinical points}

- Southern Spain is a very popular British tourist destination.

- Dogs are commonly infected by leishmania protozoa and act as the reservoir for human infection. The vector is the sandfly.

- The South American form of mucocutaneous leishmaniasis can be caused by Mediterranean species.

concentrated in macrophages and has excellent antileishmanial activity with lower toxicity than amphotericin B of antimonials. Nevertheless the infection proved very resistant to treatment. And the patient relapsed once treatment was discontinued.

Leishmania infection should be included in the differential diagnosis of tourists who perhaps, months later, develop painless cutaneous ulcers or hepatosplenomegaly.

\section{ACKNOWLEDGEMENT}

We acknowledge the assistance of Dr George Wyatt, Senior Lecturer in Tropical Medicine and Dr Nick Beeching Senior Lecturer in Infectious Diseases at the Liverpool School of Tropical Medicine for leishmania polymerase chain reaction analysis and advice on treatment.

\section{Authors' affiliations}

N Tomson, A A Moir, Department of Ear, Nose, and Throat, Leicester Royal Infirmary, Leicester

R P Symonds, Department of Oncology

C H Kendall, Department of Histopathology

M J Wiselka, Department of Infectious Disease

Correspondence to: Dr R P Symonds, Department of Oncology, University of Leicester, Leicester Royal Infirmary, Leicester LE I 5WW, UK; paul.symonds@uhl-tr.nhs.uk

Submitted 25 February 2002

Accepted 1 October 2002

\section{REFERENCES}

WHO expert committee. Control of leishmaniasis. Geneva: World Health Organisation, 1990

2 Lainson R, Shaw JJ. Evolution, classification and geographic distribution. In: Peters W, Killick-Kendrick R, eds. The leishmaniases in biology and medicine.Vol 1. Orlando: Academic Press, 1987: 1-120.

3 Lohuis PJF, Liporsky MM, Hoepelman AIM, et al. Leishmania braziliensis presenting as a granulomatous lesion in the nasal mucosa. J Laryngol presenting as a granulom
Otol 1997:111:973-5.

4 Verham GA, Sadiq H, Mallon EA. Nasal leishmaniasis. J Laryngol Otol 1993; 107:834-6

5 Khayat CM, Perreau P, Souchal-Delacour I, et al. Mucocutaneous leishmaniasis. Report of 2 cases. Ann Otolaryngol Chir Cervicofac 1990; 107:494-9

6 Borzoni F, Gradoni L, Gramiccia M, et al. A case of lingual and palatine localization of a viscerotropic Leishmania infantum zymodeme in Sardinia, Italy. Trop Med Parasitol 1991:42:193-4.

7 Miralles ES, Nunez M Hilara Y et al. Mucocutaneous leishmaniasis and HIV. Dermatology 1994;189:275-7.

Davidson RN. Practical guide for the treatment of leishmaniasis. Drugs Davidson RN. Practica.

9 Davidson RN. Visceral leishmaniasis in clinical practice. J Infect 1999;39:112-16. 